\title{
Accuracy of analytical-numerical solutions of the Michaelis-Menten equation
}

\author{
GILBERTO GONZALEZ-PARRA ${ }^{1 *}$, LUIS ACEDO ${ }^{2}$ \\ and ABRAHAM ARENAS ${ }^{3}$ \\ ${ }^{1}$ Departamento de Cálculo, Universidad de los Andes, Mérida, Venezuela \\ ${ }^{2}$ Instituto de Matemática Multidisciplinar, Universidad Politécnica de Valencia, España \\ ${ }^{3}$ Departamento de Matemáticas y Estadística, Universidad de Córdoba, Montería, Colombia \\ E-mails: gcarlos@ula.ve / luiacrod@imm.upv.es / aarenas@sinu.unicordoba.edu.co
}

\begin{abstract}
It is the aim of this paper to investigate a suitable approach to compute solutions of the powerful Michaelis-Menten enzyme reaction equation with less computational effort. We obtain analytical-numerical solutions using piecewise finite series by means of the differential transformation method, DTM. In addition, we compute a global analytical solution by a modal series expansion. The Michaelis-Menten equation considered here describes the rate of depletion of the substrate of interest and in general is a powerful approach to describe enzyme processes. A comparison of the numerical solutions using DTM, Adomian decomposition and Runge-Kutta methods is presented. The numerical results show that the DTM is accurate, easy to apply and the obtained solutions retain the positivity property of the continuous model. It is concluded that the analytic form of the DTM and global modal series solutions are accurate, and require less computational effort than other approaches thus making them more convenient.
\end{abstract}

Mathematical subject classification: Primary: 65L05; Secondary: 34A45.

Key words: Michaelis-Menten, kinetics, enzyme-catalyzed reaction, differential transformation method (DTM), modal series.

\section{Introduction}

Enzymes are proteins that catalyze all chemical reactions essential for living organisms. As any catalyst, they do not modify the products or the chemical 
equilibrium but only the velocity of these reactions. A set of several enzymes appears at every step of the basic metabolic cycle: the citric acid cycle or Krebs cycle and according to some biologists their role in these cycles was crucial in the origin of life in the so called "metabolism first" scenario [24]. Some enzymes such as $\beta$-lactamases have become the objective of extensive studies because they catalyze hydrolyzing mechanisms for antibiotics and, consequently make some bacteria such as Escherichia coli resistant against traditional treatment [25]. For these reasons, understanding the kinetics of enzyme reactions is very important from a fundamental point of view in biology and medicine. In modern science, all enzymes, pathways and whole-cell models are created, analyzed and simulated using computers. These analyses gave rise to enzyme kinetics, metabolic control analysis and pathway modeling and are now part of the field of systems biology. Simultaneous experimental (in vitro measurements), theoretical (ODE, regression analysis) and informatics (peer review journals, libraries) achievements were all required for scientific progress.

The Michaelis-Menten equation has been widely used to describe the kinetics of enzyme-catalyzed reactions. For instance in [5] it is investigated the Michaelis-Menten enzyme kinetics on low-dimensional lattices by means of Monte Carlo simulations where the formulation of the reaction scheme is modeled by evolution rules. Kinetic studies on enzymes are among the most important tools for understanding biological interactions at the molecular level. In combination with new approaches in genetic engineering and structure determination, there have been major efforts in recent years to develop more sensitive and precise techniques for characterizing the kinetics of enzyme reactions [7].

The Michaelis-Menten equation has proven to be a simple yet powerful approach to describe enzyme processes. Its power resides in the time-independent hyperbolic relation of the initial velocity with initial substrate concentration that leads to a linear double reciprocal, from which the reaction parameters, namely the rate constant $K_{m}$ and maximum velocity $V_{m}$ can be determined.

Evoking the pseudo-steady state approximation, the Michaelis-Menten equation reduces to a single first-order nonlinear ordinary differential equation which describes the rate of depletion of the substrate of interest [21]. While this equation can be readily integrated, the resulting expression is implicit in the substrate concentration. Thus, it is common to compute the substrate concentration by root-solving techniques such as the bisection and Newton-Raphson 
methods [13]. Alternatively, substrate concentration can be estimated by numerically integrating the differential form of the Michaelis-Menten equation as shown in several studies $[14,15]$. In [12] the estimation of enzyme kinetic parameters by nonlinear fitting reaction curves was compared to the integrated Michaelis-Menten rate equations. Here we are interested in improving the aforementioned methods to compute the substrate concentration in the MichaelisMenten equation. Algebraic solutions provides a simpler alternative to numerical approaches such as differential equation evaluation and root-solving techniques that are currently used [21]. An explicit closed-form solution to the MichaelisMenten equation has been proposed only recently [9]. This solution is based on the Lambert W function [11]. Having an accurate closed-form solution to the Michaelis-Menten equation opens up the possibility of examining the utility of other non-conventional solution techniques to solve the Michaelis-Menten equation.

In order to improve the computation of the substrate concentration in the Michaelis-Menten equation we rely on DTM and a global modal series. Starting from a differential equation system with initial conditions, the DTM leads to a system of algebraic equations whose solutions are the coefficients of a series solution. In this work the DTM is numerically compared with the multistage Adomian decomposition, Runge-Kutta method and the global modal series. The classical Adomian method has been used in the past to obtain solutions to the Michaelis-Menten ordinary differential equation [16, 17, 18]. In [21] the Adomian decomposition with domain split has been applied successfully to the Michaelis-Menten ordinary differential equation.

It is important to remark that numerical comparisons are made regarding two issues: accuracy and computation time. Computation time is important since in several cases the kinetic parameters are estimated by minimizing the residual sum of squares error between experimental and calculated substrate concentration data $[21,13,12,20]$. Thus a faster computation time will improve overall estimating procedure time [21]. It should be mentioned that analytic forms are obtained with the DTM, Adomian and modal series methods. However, with Runge-Kutta methods and nonlinear solvers only discrete solutions are obtained.

The organization of this paper is as follows. In Section 2, basic definitions and properties of the differential transform method are presented. In Section 3 the DTM is applied to the Michaelis-Menten ordinary differential equation. Sec- 
tion 4 is devoted to the application of the modal series in order to obtain a global solution. In Section 5 comparisons between DTM, the fourth-order Runge-Kutta (RK4) method, multistage Adomian method and a nonlinear solver based on the Adomian method are included. Finally in Section 6 conclusions are presented.

\section{Basic definitions and properties of differential transformation method}

Given a system of differential equations and related initial conditions, these are transformed into a system of recurrence equations that finally leads to a system of algebraic equations whose solutions are the coefficients of a power series solution. The numerical solution of the system of differential equation in the time domain can be obtained in the form of a finite-term series in terms of a chosen basis system. The advantage of this method is that it is not necessary to do linearization or perturbations. Furthermore, large computational work and round-off errors are avoided. For the sake of clarity in the presentation of the DTM and in order to help to the reader we summarize the main issues of the method that can be found in [8].

Definition 1.1. Let $x(t)$ be analytic in the time domain $D$, and if $X(k)$ is defined as

$$
X(k)=M(k)\left[\frac{d^{k} x(t)}{d t^{k}}\right]_{t=t_{i}}
$$

where $k \in \mathbb{Z}^{+} \cup\{0\}$, then the function $x(t)$ can be described as

$$
x(t)=\frac{1}{q(t)} \sum_{k=0}^{\infty} \frac{\left(t-t_{i}\right)^{k}}{k !} \frac{X(k)}{M(k)},
$$

where $M(k) \neq 0$ and $q(t) \neq 0$. $M(k)$ is the weighting factor and $q(t)$ is regarded as a kernel corresponding to $x(t)$.

Definition 1.2. Let $[0, H]$ the interval of simulation with $H$ the time horizon of interest. We take a partition of the interval $[0, H]$ as $\left\{0=t_{0}, t_{1}, \ldots, t_{n}=H\right\}$ such that $t_{i}<t_{i+1}$ and $H_{i}=t_{i+1}-t_{i}$ for $i=0, \ldots, n$. Let $M(k)=\frac{H_{i}^{k}}{k !}$, $q(t)=1$ and $x(t)$ be a analytic function in $[0, H]$. It then defines the differential transformation as

$$
X(k)=\frac{H_{i}^{k}}{k !}\left[\frac{d^{k} x(t)}{d t^{k}}\right]_{t=t_{i}} \text { where } k \in \mathbb{Z}^{+} \cup\{0\},
$$


and its differential inverse transformation of $X(k)$ is defined as follow

$$
x(t)=\sum_{k=0}^{\infty}\left(\frac{t}{H_{i}}\right)^{k} X(k), \text { for } t \in\left[t_{i}, t_{i+1}\right] .
$$

In order to obtain a globally accurate solution we divide the time domain $H$ in $n$ subdomains. In this way, the differential equation can then be solved in each subdomain to obtain a piecewise finite series solution $[6,3,2]$. The main advantage of domain split process is that only a few series terms are required to construct the solution in a small time interval $H_{i}$, where $H=\sum_{i=1}^{n} H_{i}$. Considering the function $x(t)$ in the $i$-th sub-domain $\left(t_{i-1} \leq t \leq t_{i}\right)$, the one dimensional differential transformation is given by

$$
x(t)=\sum_{k=0}^{n}\left(\frac{t}{H_{i}}\right)^{k} X_{i-1}(k), \text { where } X_{i-1}(0)=x_{i-1}\left(t_{i-1}\right) .
$$

The operation properties of the differential transformation. We consider $q(t)=1, M(k)=\frac{H_{i}^{k}}{k !}$ and $x^{1}(t), x^{2}(t)$ two uncorrelated functions of time $t$. Let $\mathcal{D}$ be the differential transformation operator. Thus, $X^{i}(k)=\mathcal{D}\left[x^{i}(t)\right]$. The properties of the differential transformation can be seen in Table 1.

\begin{tabular}{|c|c|}
\hline Original function & Transformed functions \\
\hline $\mathcal{D}\left[c_{1} x^{1}(t) \pm c_{2} x^{2}(t)\right]$ & $c_{1} X^{1}(k) \pm c_{2} X^{2}(k)$ \\
$\mathcal{D}[c]$ & $c \delta(k)$ \\
$\mathcal{D}\left[\frac{d x^{1}(t)}{d t}\right]$ & $\frac{(k+1)}{H_{i}} X^{1}(k+1)$ \\
& $\frac{X^{1}(k)-\sum_{l=0}^{k-1} Z(l) X^{2}(k-l)}{X^{2}(0)}$ \\
$\mathcal{D}\left[z(t)=x^{1}(t) / x^{2}(t)\right]$ & $\frac{}{}$ \\
\hline
\end{tabular}

Table 1 - Basic operations for differential transformation method.

\section{Solution of Michaelis-Menten equation by DTM}

In this section, the differential transformation technique is applied to solve the Michaelis-Menten ordinary differential equation. The Michaelis-Menten equation (6) considered here describes the rate of depletion of the substrate of interest.

$$
\dot{s}(t)=-\frac{V_{m} s(t)}{K_{m}+s(t)},
$$


where $s(t)$ is the substrate concentration, and $V_{m}$ and $K_{m}$ are the limiting rate and Michaelis constant, respectively.

In the next Section numerical simulations are performed with $V_{m}=1, K_{m}=1$ and initial condition $s_{0}=10$. However, in some simulations these values are modified but are clearly mentioned. From the properties given in Section (1), the corresponding spectrum for system (6) can be determined as one recurrence system given by,

$$
\mathbf{S}(k+1)=-\frac{V_{m} H_{i} \mathbf{Z}(k)}{k+1},
$$

where $Z(k)$ is the spectrum of $z(t)=-\frac{s(t)}{K_{m}+s(t)}$, given by

$$
\mathbf{Z}(k)=\frac{\mathbf{S}(k)+\sum_{l=0}^{k-1} \mathbf{Z}(l)\left(K_{m} \delta(k-l)+\mathbf{S}(k-l)\right)}{K_{m} \delta(0)+\mathbf{S}(0)}, k \geq 1,
$$

with

$$
\mathbf{Z}(0)=\frac{\mathbf{S}(0)}{K_{m} \delta(0)+\mathbf{S}(0)},
$$

where the initial condition is $\mathbf{S}(0)=s(0)$. Thus, from a process of inverse differential transformation, the solutions can be obtained on each sub-domain taking $n+1$ terms for the power series like equation (5), i.e.,

$$
s_{i}(t)=\sum_{k=0}^{n}\left(\frac{t}{H_{i}}\right)^{k} \mathbf{S}(k), 0 \leq t \leq H_{i},
$$

provided that the solution holds with:

$$
s(t)=\sum_{i=0}^{n} s_{i}(t)
$$

\section{Solution of Michaelis-Menten equation by modal series}

Here we obtain a global analytical solution by the modal series which avoids the split of time domain in subintervals and produces accurate solutions. This method is based on the following modal expansion series:

$$
s(t)=\sum_{k=0}^{\infty} \mathcal{A}_{k} e^{-k \omega t}, \omega>0,
$$


where the frequencies and the coefficients $\mathcal{A}_{k}, k=2,3 \ldots$ are determined by a recurrence relation in terms of $\mathcal{A}_{1}$ and the parameters of the model. For more details concerning this method, refer to [4]. Indeed, equation (6) can be rewritten in the following form

$$
K_{m} \dot{s}(t)+s(t) \dot{s}(t)=-V_{m} s(t) .
$$

Now, from (12) it follows that

$$
\dot{s}(t)=-\sum_{k=1}^{\infty} k \omega \mathcal{A}_{k} e^{-k \omega t}=-\sum_{k=0}^{\infty} k \omega \mathcal{A}_{k} e^{-k \omega t},
$$

and using the Cauchy product we obtain

$$
s(t) \dot{s}(t)=-\sum_{k=0}^{\infty} e^{-k \omega t}\left(\sum_{j=0}^{k} \omega j \mathcal{A}_{j} \mathcal{A}_{k-j}\right) .
$$

Inserting (12), (14) and (15) into (13) one obtains

$$
\sum_{k=0}^{\infty}\left(-K_{m} k \omega \mathcal{A}_{k}-\sum_{j=0}^{k} j \omega \mathcal{A}_{j} \mathcal{A}_{k-j}+V_{m} \mathcal{A}_{k}\right) e^{-k \omega t}=0 .
$$

Thus, one gets that

$$
-K_{m} k \omega \mathcal{A}_{k}-\sum_{j=0}^{k} j \omega \mathcal{A}_{j} \mathcal{A}_{k-j}+V_{m} \mathcal{A}_{k}=0, \text { for } k=0,1, \ldots,
$$

where we have taken into account that $e^{-k \omega t}(k=0,1, \ldots)$ are a linearly independent base of exponential functions. It is clear that $\mathcal{A}_{0}=0$, and if we assume that $\mathcal{A}_{1} \neq 0$, then we have that

$$
-K_{m} \omega \mathcal{A}_{1}-\omega \mathcal{A}_{1} \mathcal{A}_{0}+V_{m} \mathcal{A}_{1}=0,
$$

i.e., $\omega=V_{m} / K_{m}$. Therefore, we can establish a recurrence formula for $\mathcal{A}_{k}$ as follows

$$
\mathcal{A}_{k}=\frac{\omega \sum_{j=1}^{k-1} j \mathcal{A}_{j} \mathcal{A}_{k-j}}{V_{m}-k \omega K_{m}}, \text { for } k \geq 2,
$$


where $\mathcal{A}_{1} \neq 0$ is chosen suitably. The initial condition is related with the $\mathcal{A}_{k} s$ by

$$
s(0)=\sum_{k=1}^{\infty} \mathcal{A}_{k} .
$$

Moreover, if we put $\mathcal{B}_{1}=\left|\mathcal{A}_{1}\right|$, and $\mathcal{B}_{k}=\left|\mathcal{A}_{k}\right|$ for $k \geq 2$, we obtain the following expression to $\mathcal{B}_{k}=\frac{1}{K_{m}(k-1)} \sum_{j=1}^{k-1} j \mathcal{B}_{j} \mathcal{B}_{k-j}$. Thus, one gets the recurrence relation

$$
\begin{aligned}
& \frac{\mathcal{B}_{2}}{\mathcal{B}_{1}}=\frac{1}{1} \frac{\mathcal{B}_{1}}{K_{m}}=\frac{(1+1)^{0}}{1^{0}} \frac{\mathcal{B}_{1}}{K_{m}}, \frac{\mathcal{B}_{3}}{\mathcal{B}_{2}}=\frac{3}{2} \frac{\mathcal{B}_{1}}{K_{m}}=\frac{(1+2)^{1}}{2^{1}} \frac{\mathcal{B}_{1}}{K_{m}}, \\
& \frac{\mathcal{B}_{4}}{\mathcal{B}_{3}}=\frac{16}{9} \frac{\mathcal{B}_{1}}{K_{m}}=\frac{(1+3)^{2}}{3^{2}} \frac{\mathcal{B}_{1}}{K_{m}}, \frac{\mathcal{B}_{5}}{\mathcal{B}_{4}}=\frac{125}{64} \frac{\mathcal{B}_{1}}{K_{m}}=\frac{(1+4)^{3}}{4^{3}} \frac{\mathcal{B}_{1}}{K_{m}}, \\
& \frac{\mathcal{B}_{6}}{\mathcal{B}_{5}}=\frac{1296}{625} \frac{\mathcal{B}_{1}}{K_{m}}=\frac{(1+5)^{4}}{5^{4}} \frac{\mathcal{B}_{1}}{K_{m}}, \frac{\mathcal{B}_{7}}{\mathcal{B}_{6}}=\frac{16807}{7776} \frac{\mathcal{B}_{1}}{K_{m}}=\frac{(1+6)^{5}}{6^{5}} \frac{\mathcal{B}_{1}}{K_{m}}, \\
& \frac{\mathcal{B}_{8}}{\mathcal{B}_{7}}=\frac{262144}{117649} \frac{\mathcal{B}_{1}}{K_{m}}=\frac{(1+7)^{6}}{7^{6}} \frac{\mathcal{B}_{1}}{K_{m}}, \cdots \\
& \frac{\mathcal{B}_{k+1}}{\mathcal{B}_{k}}=\left(1+\frac{1}{k}\right)^{k-1} \frac{\mathcal{B}_{1}}{K_{m}}=\left(1+\frac{1}{k}\right)^{k}\left(\frac{k}{1+k}\right) \frac{\mathcal{B}_{1}}{K_{m}} .
\end{aligned}
$$

If we choose $\mathcal{B}_{1}=\left|\mathcal{A}_{1}\right| \leq \frac{K_{m}}{e}$, then $\mathcal{B}_{k}$ is a monotone decreasing sequence, and the series $\sum_{k=1}^{\infty}(-1)^{k} \mathcal{B}_{k}$ converges absolutely. Now, if $k$ is even, we have that

$$
\mathcal{B}_{k+1}=\frac{k+1}{k} \frac{1}{K_{m}}\left\{\mathcal{B}_{1} \mathcal{B}_{k}+\mathcal{B}_{2} \mathcal{B}_{k-1}+\cdots+\mathcal{B}_{\frac{k-1}{2}} \mathcal{B}_{\frac{k+3}{2}}+\mathcal{B}_{\frac{k+1}{2}}^{2}\right\} .
$$

Since $\mathcal{B}_{k}<\mathcal{B}_{k-1}<\mathcal{B}_{k-2}<\cdots$, then $\mathcal{B}_{k+1}$ is lower bounded as follows

$$
\mathcal{B}_{k+1}>\frac{k+1}{k} \frac{1}{K_{m}} \sum_{j=1}^{\frac{k+1}{2}} \mathcal{B}_{j} .
$$

Suppose that $\sum_{k=1}^{\infty} \mathcal{B}_{k}>K_{m}$, then there exists $N$ such that $\sum_{k=1}^{\frac{N+1}{2}} \mathcal{B}_{k}>K_{m}+\epsilon$, where $\epsilon>0$. Therefore,

$$
\frac{\mathcal{B}_{N+1}}{\mathcal{B}_{N}}>\frac{N+1}{N} \frac{K_{m}+\epsilon}{K_{m}}>1 .
$$


This contradicts the hypothesis of the statement. Thus, $\sum_{k=1}^{\infty} \mathcal{B}_{k}<K_{m}$. The above analysis allows us to state the following theorem:

Theorem 3.1. If $\mathcal{B}_{1}=\left|\mathcal{A}_{1}\right| \leq \frac{K_{m}}{e}$, and $\mathcal{B}_{k}=\left|\mathcal{A}_{k}\right|$ for $k \geq 2$, then the series $\sum_{k=1}^{\infty}(-1)^{k} \mathcal{B}_{k}$ converges absolutely, and furthermore $\sum_{k=1}^{\infty} \mathcal{B}_{k}<K_{m}$.

Notice that in this case $\mathcal{A}_{0}=0$ since $\lim _{t \rightarrow \infty} s(t)=0$.

\section{Numerical results}

In this section, numerical comparisons between DTM, multistage Adomian and Runge-Kutta methods are performed in order to investigate the accuracy and computation time of each method. Also, we present the numerical results of the global analytical solution obtained by the modal series in order to show its efficiency. Since an explicit closed-form solution to the Michaelis-Menten equation based on the Lambert $\mathrm{W}$ function is available we can examine the absolute errors of each one of the methods used to solve the Michaelis-Menten equation. The explicit closed-form solution to the Michaelis-Menten equation is:

$$
s(t)=K_{m} W\left(\frac{s_{0}}{K_{m}} \exp \left[\frac{s_{0}-V_{m} t}{K_{m}}\right]\right),
$$

where $\mathrm{W}$ is the Lambert function [11].

First of all in order to illustrate the type of solutions that are obtained with the multistage Adomian method (MADM) and the DTM, we present Tables 2 and 3. In Table 2 it can be seen the piecewise finite series solution obtained with the multistage Adomian method for the Michaelis-Menten ordinary differential equation in the time interval $[0,5]$ with a time step size $h=1$. Table 3 presents an example of the piecewise finite series solution obtained with the DTM for the Michaelis-Menten ordinary differential equation.

In Figure 1 a comparison of the numerical solutions of the 4-term DTM with RK4 methods for Michaelis-Menten differential equation (6) is shown. It can be seen that the DTM reproduces the correct behavior of the solution for the Michaelis-Menten ordinary differential equation. Notice that for a time step size $h=2.8$ the well known Runge-Kutta method fails and DTM gives the correct solution for the same time step size. However, in Figure 2 it can be observed that both methods give accurate solutions. 


\begin{tabular}{|c|c|}
\hline Time & MADM \\
\hline $0.0 \leq t<1.0$ & $10.0-0.909 t+0.0037 t^{2}+0.00019 t^{3}+0.000011 t^{4}$ \\
$1.0 \leq t<2.0$ & $10.0-0.909 t+0.0037 t^{2}+0.00018 t^{3}+0.000015 t^{4}$ \\
$2.0 \leq t<3.0$ & $10.0-0.909 t+0.0038 t^{2}+0.00015 t^{3}+0.00002 t^{4}$ \\
$3.0 \leq t<4.0$ & $10.0004-0.909 t+0.0041 t^{2}+0.000067 t^{3}+0.000029 t^{4}$ \\
$4.0 \leq t<5.0$ & $10.002-0.912 t+0.0051 t^{2}-0.00011 t^{3}+0.000042 t^{4}$ \\
\hline
\end{tabular}

Table 2 - Piecewise finite series approximate solution obtained with the multistage Adomian method at different time intervals with $V_{m}=1, K_{m}=1$.

\begin{tabular}{|c|c|}
\hline Time & DTM \\
\hline $0.0 \leq t<1.0$ & $10.0-0.909 t+0.0071 t^{2}+0.00037 t^{3}+0.00002 t^{4}$ \\
$1.0 \leq t<2.0$ & $10.003-0.909 t+0.0037 t^{2}+0.00018 t^{3}+0.000015 t^{4}$ \\
$2.0 \leq t<3.0$ & $10.003-0.909 t+0.0038 t^{2}+0.00015 t^{3}+0.00002 t^{4}$ \\
$3.0 \leq t<4.0$ & $10.004-0.909 t+0.0041 t^{2}+0.000067 t^{3}+0.000029 t^{4}$ \\
$4.0 \leq t<5.0$ & $10.006-0.912 t+0.0051 t^{2}-0.00011 t^{3}+0.000042 t^{4}$ \\
\hline
\end{tabular}

Table 3 - Piecewise finite series approximate solution obtained with the DTM at different time intervals with $V_{m}=1, K_{m}=1$.

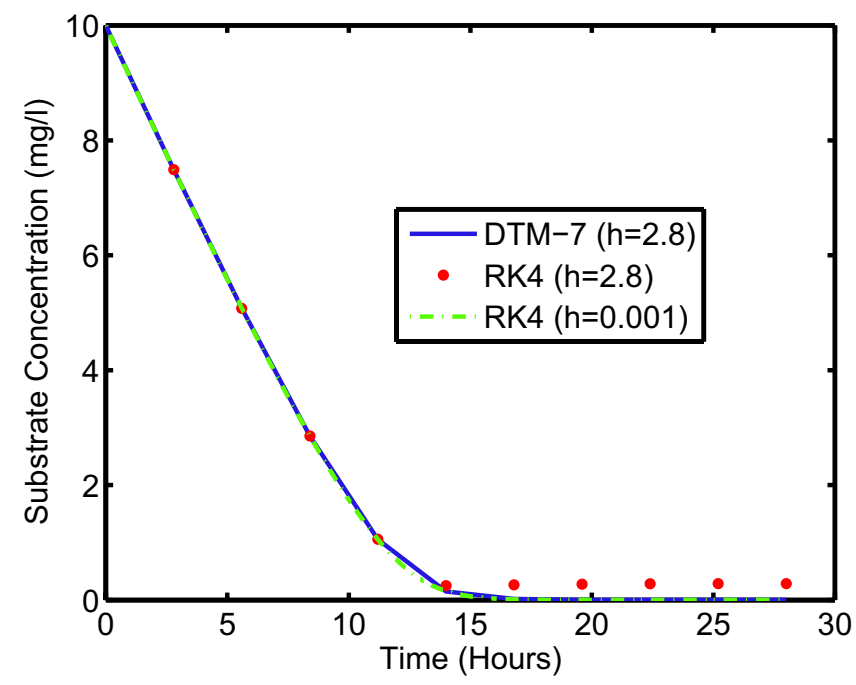

Figure 1-Comparison of the numerical solutions of the 4-term DTM with $R K 4$ methods for Michaelis-Menten differential equation (6). 


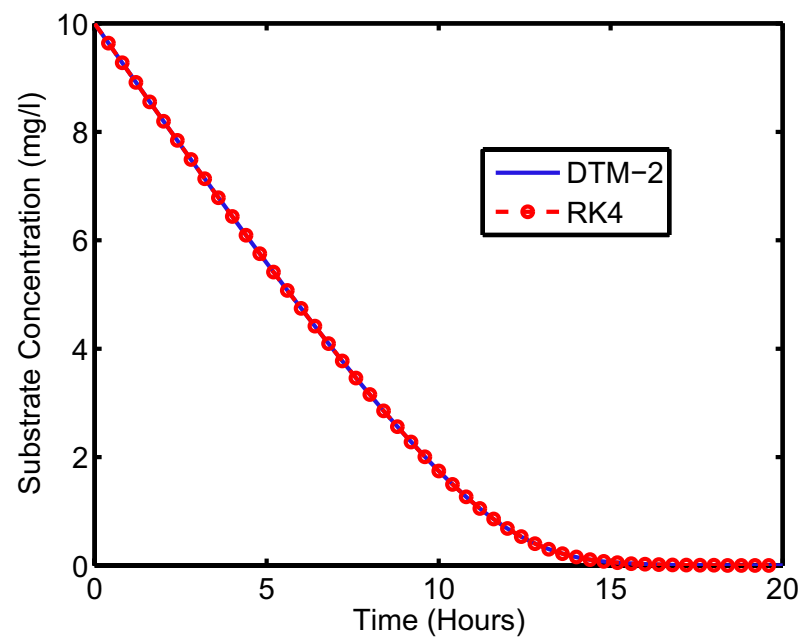

Figure 2-Comparison of the numerical solutions of the 4-term DTM with $R K 4$ methods with $h=0.2$ for Michaelis-Menten differential equation (6).

In Tables 4 and 5 we present the absolute errors for the 2-term DTM solution, 2-term multistage Adomian method and the second-order Runge-Kutta (RK2) solution when a time step size of $h=0.2$ is used in all methods. These results show the numerical consistency of the DTM. Table 6 shows the absolute errors of the aforementioned methods but now with 4-terms for the DTM and multistage Adomian methods, and the fourth-order Runge-Kutta (RK4) solution. Notice that in Tables 6 and 7 the numerical results for DTM and multistage Adomian have similar accuracy. Therefore, the more convenient method must be the one with less computation time. In Table 7 we present the errors of the DTM with different number of terms. Notice, that as expected the accuracy of the solution is increased when more terms are implemented. This approach is easy to be implemented since the code is totally reusable. However, for increasing the order of Runge-Kutta method it is necessary to implement a new code.

In Table 8 we present the computation time required to obtain an error of approximately $10^{-5}$ at different times using DTM $(h=0.2)$ and multistage Adomian method. Computation time is important because in order to estimate the parameters $V_{m}$ and $K_{m}$ through synthetic substrate concentration data it is necessary to solve the Michaelis-Menten ordinary differential equation several times to minimize the residual sum of squares error between synthetic and calculated substrate concentration data. 


\begin{tabular}{|c|c|c|c|}
\hline Time & DTM & $R K 2$ & $M A D M$ \\
\hline 0.2 & $0.34 E-04$ & $0.93 E-06$ & $0.15 E-05$ \\
0.6 & $0.48 E-04$ & $0.29 E-05$ & $0.49 E-05$ \\
1.0 & $0.61 E-04$ & $0.51 E-05$ & $0.87 E-05$ \\
5.0 & $0.30 E-03$ & $0.48 E-04$ & $0.76 E-04$ \\
10.0 & $0.18 E-02$ & $0.36 E-03$ & $0.43 E-03$ \\
\hline
\end{tabular}

Table 4 - Comparison of the errors between the 2-term DTM $\left(H_{i}=0.2\right)$ with $R K 2(h=0.2)$ and 2-term $\operatorname{MADM}(h=0.2)$ methods for Michaelis-Menten differential equation (6) with $K_{m}=1$.

\begin{tabular}{|c|c|c|c|}
\hline Time & DTM & $R K 2$ & $M A D M$ \\
\hline 0.2 & $0.16 E-07$ & $0.91 E-08$ & $0.66 E-08$ \\
0.6 & $0.44 E-07$ & $0.27 E-07$ & $0.19 E-07$ \\
1.0 & $0.72 E-07$ & $0.45 E-07$ & $0.32 E-07$ \\
5.0 & $0.34 E-06$ & $0.22 E-06$ & $0.16 E-06$ \\
10.0 & $0.64 E-06$ & $0.42 E-06$ & $0.31 E-06$ \\
\hline
\end{tabular}

Table 5 - Comparison of the errors between the 2-term DTM $\left(H_{i}=0.2\right)$ with $R K 2(h=0.2)$ and 2-term $M A D M(h=0.2)$ methods for Michaelis-Menten differential equation (6) with $K_{m}=100$.

\begin{tabular}{|c|c|c|c|}
\hline Time & DTM & $R K 4$ & $M A D M$ \\
\hline 0.2 & $0.21 E-9$ & $0.12 E-10$ & $0.21 E-9$ \\
0.6 & $0.67 E-9$ & $0.41 E-10$ & $0.67 E-9$ \\
1.0 & $0.12 E-8$ & $0.75 E-10$ & $0.12 E-8$ \\
5.0 & $0.15 E-7$ & $0.11 E-8$ & $0.15 E-7$ \\
10.0 & $0.84 E-7$ & $0.34 E-7$ & $0.84 E-7$ \\
\hline
\end{tabular}

Table 6 - Comparison of the errors between the 4-term DTM $\left(H_{i}=0.2\right)$ with $R K 4(h=0.2)$ and 4-term $M A D M(h=0.2)$ methods for Michaelis-Menten differential equation (6).

As it can be seen in Table 8 the DTM is faster than the multistage Adomian method since it does not require heavy symbolic computation. However, the Runke-Kutta methods require less computation time in comparison with DTM and multistage Adomian. In [21] nonlinear solvers have been used as another approach to numerically obtain the solution of Michaelis-Menten ordinary dif- 


\begin{tabular}{|c|c|c|c|}
\hline Time & 4-term DTM & 6-term DTM & 8-term DTM \\
\hline 0.2 & $0.21 E-9$ & $0.29 E-13$ & $0.37 E-17$ \\
0.6 & $0.67 E-9$ & $0.96 E-13$ & $0.12 E-16$ \\
1.0 & $0.12 E-8$ & $0.17 E-12$ & $0.23 E-16$ \\
5.0 & $0.15 E-7$ & $0.29 E-11$ & $0.35 E-15$ \\
10.0 & $0.84 E-7$ & $0.97 E-10$ & $0.68 E-13$ \\
\hline
\end{tabular}

Table 7 - Comparison of the numerical results using DTM with different number of terms and time step size $H_{i}=0.2$ for Michaelis-Menten differential equation (6).

\begin{tabular}{|c|c|c|}
\hline Time & DTM & $M A D M$ \\
\hline 1.0 & 0.01 & 0.34 \\
5.0 & 0.05 & 1.05 \\
10.0 & 0.10 & 2.19 \\
20.0 & 0.14 & 3.80 \\
60.0 & 0.51 & 10.85 \\
\hline
\end{tabular}

Table 8 - Comparison of computation time of the DTM $(h=0.2)$ and multistage Adomian method $(h=0.2)$ required to obtain an error of $10^{-5}$ at different times.

\begin{tabular}{|c|c|c|c|c|}
\hline Time & DTM & $R K 4$ & $M A D M$ & Modal \\
\hline 0.2 & $0.37465 E-8$ & $0.52 E-8$ & $0.37 E-8$ & $0.7674 E-6$ \\
0.6 & $0.78148 E-8$ & $0.15 E-7$ & $0.11 E-7$ & $0.7441 E-6$ \\
1.0 & $0.26161 E-7$ & $0.23 E-7$ & $0.17 E-7$ & $0.1790 E-6$ \\
5.0 & $0.89055 E-7$ & $0.34 E-7$ & $0.58 E-8$ & $0.1039 E-7$ \\
10.0 & $0.14229 E-7$ & $0.72 E-8$ & $0.29 E-8$ & $0.2818 E-8$ \\
\hline
\end{tabular}

Table 9 - Comparison of the errors between the 4-term DTM $\left(H_{i}=0.2\right)$ with $R K 4(h=0.2)$, 4-term $\operatorname{MADM}(h=0.2)$ and modal series (50-term) methods for Michaelis-Menten differential equation (6) with $V_{m}=0.5, K_{m}=1$ and initial condition $s_{0}=0.236755$.

ferential equation. The authors found that this approach requires a significantly higher number of computations. In addition, it is important to remark that for the nonlinear solvers an initial guess point or interval is needed a priori. If the initial guess point is far from the true solution, convergence may not be achieved.

Numerical results show that DTM is an accurate approach without long 
computation time to obtain solutions of the Michaelis-Menten ordinary differential equation. The DTM numerical solutions are comparable with the RungeKutta solutions and their accuracy can be easily improved without a new code implementation. In addition, the method has the advantage of giving a functional form of the solution within each time interval. Therefore, no further computation is necessary if the substrate depletion data is available for different sets where the experimental observations are not equal. This is not possible in purely numerical techniques like the Runge-Kutta method, which provides solution only at discrete times.

Finally, the Table 9 shows the absolute errors of the 4-term DTM $\left(H_{i}=\right.$ $0.2)$ with $R K 4(h=0.2)$, 4-term $M A D M(h=0.2)$ and modal series (50term) methods for Michaelis-Menten differential equation (6) with $V_{m}=0.5$, $K_{m}=1$ and initial condition $s_{0}=0.236755$. These results show the accuracy of the modal series expansion method which gives a global analytical solution. It is important to remark that the computation of the modal series can be done easily with a symbolic computer package and it is done only one time in contrast with the multistage Adomian method and the DTM which are applied in a sequence of time intervals. In this way this method is promising regarding computation time.

\section{Discussion and Conclusions}

In this paper piecewise finite series approximate solutions of the MichaelisMenten ordinary differential equation using the differential transformation method DTM, and modal series method has been obtained. The MichaelisMenten equation considered here describes the rate of depletion of the substrate of interest. The time domain has been split in subintervals and the approximating solutions are obtained in a sequence of time intervals in order to obtain accurate solutions. However, the accuracy can be increased easily by means of additional new terms. Starting from a differential equation system with initial conditions, the DTM leads to a system of algebraic equations whose solutions are the coefficients of a series solution. Here we compare the effectiveness of DTM with multistage Adomian and Runge-Kutta methods. The DTM solutions shown an excellent agreement with those obtained by the Runge-Kutta methods and with the analytical solution. 
In addition, we obtain a global analytical solution by a modal series expansion which avoids the split of time domain in subintervals and produces accurate solutions. However, this method is restricted to certain initial conditions. In particular, we have proven the convergence of the modal series for $s_{0}<K_{m}$ but, in general, divergent series are found for larger initial concentrations, $s(0)$. This is the major drawback of the modal series method. Future work includes the investigation of the feasibility of the extension of the modal series expansion method to any initial condition.

The numerical results show that the DTM is accurate, easy to apply, and suggest that the approximate solutions preserve the positivity property of the Michaelis-Menten ordinary differential equation. Furthermore, high accuracy can be obtained without using long computation. Moreover, the DTM does not compute the derivatives or integrals symbolically and this gives advantages over other methods such Taylor, power series or Adomian method. Finally, the analytic form of the DTM solution and its relatively high accuracy make this a competitive approach to solve the Michaelis-Menten equation and can be used for estimating the parameters $V_{m}$ and $K_{m}$ through minimizing the residual sum of squares error between experimental and calculated substrate concentration data.

Acknowledgements. Authors are grateful to the reviewers for their helpful comments and suggestions. Luis Acedo acknowledges financial support from the grant PAID-06-09 ref: 2588 of the Universitat Politècnica de València, Spain.

\section{REFERENCES}

[1] O.E. Aiéllo, V.J. Haas, M.A.A. da Silva and A. Caliri, Solution of deterministic stochastic epidemic models by dynamical Monte Carlo method. Physical Review E Physica A: Statistical Mechanics and its Applications, 282 (2000), 546-558.

[2] Abraham J. Arenas, Gilberto González-Parra and Benito M. Chen-Charpentier, Dynamical analysis of the transmission of seasonal diseases using the differential transformation method. Mathematical and Computer Modelling, 50(5-6) (2009), 765-776.

[3] Hsin-Ping Chu and Chieh-Li Chen, Hybrid differential transform and finite difference method to solve the nonlinear heat conduction problem. Communications in Nonlinear Science and Numerical Simulation, 13(8) (2008), 1605-1614. 
[4] L. Acedo, Gilberto González-Parra and Abraham J. Arenas, An exact global solution for the classical SIRS epidemic model. Nonlinear Analysis: Real World Applications, 11(3) (2010), 1819-1825.

[5] Hugues Berry, Monte Carlo Simulations of Enzyme Reactions in Two Dimensions: Fractal Kinetics and Spatial Segregation. Biophysical Journal, 83(4) (2002), 1891-1901.

[6] C.L. Chen, S.H. Lin and C.K. Chen, Application of Taylor transformation to nonlinear predictive control problem. Appl. Math. Model., 20 (1996), 699-710.

[7] U. Kettling, A. Koltermann, P. Schwille and M. Eigen, Real-time enzyme kinetics monitored by dual-color fluorescence cross-correlation spectroscopy. Proceedings of the National Academy of Sciences of the United States of America, 95(4) (1998), 1416-1420.

[8] J.K. Zhou, Differential Transformation and its Applications for Electrical Circuits. Huazhong University Press, Wuhan, (in Chinese) (1986).

[9] S. Schnell and C. Mendoza, Closed form solution for time-dependent enzyme kinetics spectroscopy. Journal of Theoretical Biolog, 187 (1997), 207-212.

[10] L. Michaelis and M. L. Menten, Die kinetik der invertinwirkung. Biochemistry Z, 49 (1913), 333-369.

[11] R.M. Corless, G.H. Gonnet, D.E. Hare, D.J. Jeffrey and D.E. KnuthL, On the Lambert $W$ function. Advances in Computational Mathematics, 5 (1996), 329359.

[12] Fei Liao, Xiao-Yun Zhu, Yong-Mei Wang and Yu-Ping Zuo, The comparison of the estimation of enzyme kinetic parameters by fitting reaction curve to the integrated Michaelis-Menten rate equations of different predictor variables. Journal of Biochemical and Biophysical Methods, 62(1) (2005), 13-24.

[13] R.G. Duggleby, Analysis of enzyme progress curves by non-linear regression. Methods in Enzymology, 249 (1995), 61-90.

[14] R.G. Duggleby, Quantitative analysis of the time course of enzyme catalyzed reactions. Methods in Enzymology, 24 (2001), 168-174.

[15] C.T. Zimmerle and C. Frieden, Analysis of progress curves by simulations generated by numerical integration. Biochemical Journal, 258 (1989), 381-387.

[16] B.T. Bullman and P.W. Kuchel, Comparison of biochemical simulations using integrators derived from "Adomian" decomposition with traditional methods. Biomedica Biochimica Acta, 8/9 (1990), 661-670. 
[17] A. Sen, An application of the Adomian decomposition method to the transient behavior of a model biochemical reaction. Journal of Mathematical Analysis and Applications, 131 (1988), 232-245.

[18] A. Sen, An approximate solution for the transient behavior of enzyme-catalyzed reactions: The irreversible Michaelis-Menten kinetics. Mathematical Biosciences, 85 (1987), 141-151.

[19] A. Sen, On the time course of the reversible Michaelis-Menten reaction. Journal of Theoretical Biology, 135 (1988), 483-493.

[20] C.T. Goudar, J.R. Sonnad and R.G. Duggleby, Parameter estimation using a direct solution of the integrated Michaelis-Menten equation. Biochimica et Biophysica Acta, 1429 (1989), 377-383.

[21] J.R. Sonnad and C.T. Goudar, Solution of the Michaelis-Menten equation using the decomposition method. Mathematical Biosciences and Engineering, 6 (2009), $173-188$.

[22] G. Adomian, Solving frontier problems of physics: the decomposition method. Boston, Kluwer Academic Publishers (1994).

[23] A. Repaci, Nonlinear dynamical systems: On the accuracy of Adomian's decomposition method. Appl. Math. Lett., 3(4) (1990), 35-39.

[24] Addy Pross, Causation and the origin of life, metabolism or replication first? Origins of Life and Evolution of the Biosphere, Kluwer Academic Publishers, 34 (2004), 307-321.

[25] D.L Paterson and R.A Bonomo, Extended-spectrum $\beta$-lactamases: a clinical update. Clin. Microbiol Rev., 18(4) (2005), 657-686. 